\title{
MUJERES MIGRANTES, VULNERABILIDAD Y DERECHOS HUMANOS
}

\author{
MIGRANT WOMEN, VULNERABILITY AND HUMAN RIGHTS
}

\author{
TERESA REBOLLEDO GÁMEZ* \\ ROCÍO RODRÍGUEZ CASADO**
}

\begin{abstract}
RESUMEN
Las transformaciones sociales, culturales y económicas acaecidas en las últimas décadas han implicado cambios en el fenómeno migratorio. Entre estos cambios se encuentra la feminización de las migraciones, entendida como un incremento del número de mujeres que participan de los procesos migratorios, pero también la presencia de nuevos roles en los que las mujeres se han convertido en líderes de sus proyectos de viaje. En este trabajo se pretende analizar el incremento y la implicación de las mujeres en los actuales procesos migratorios, con el fin de visibilizar la importancia de la perspectiva de género en estudios y acciones políticas para dar respuesta a esta situación de riesgo y vulnerabilidad agravada por el hecho de ser mujer.
\end{abstract}

PALABRAS CLAVE: migración, género, derechos humanos, vulnerabilidad.

\begin{abstract}
The social, cultural and economic transformations that have occurred in recent decades have involved changes in the migration phenomenon. Among these changes is the feminization of migrations, understood as an increase in the number of women who participate in migration processes, but also the presence of new roles in which women have become leaders in their travel projects. This paper aims to analyze the increase and involvement of women in the current migration processes, in order to make visible the importance of the gender perspective in studies and political actions to respond to this situation of risk and vulnerability aggravated by the being a woman.
\end{abstract}

KEYWORDS: migration, gender, human rights, vulnerability.

* Doctora en Desarrollo y Ciudadanía. Facultad de Ciencias Sociales. Universidad Pablo de Olavide. Carretera de Utrera, km. 1. CP. 41013. Sevilla (España).

E-mail address: tjrebgam@upo.es.

** Doctora en Desarrollo y Ciudadanía. Facultad de Ciencias Sociales. Universidad Pablo de Olavide. Carretera de Utrera, km. 1. CP. 41013. Sevilla (España).

E-mail address: mrrodcas@upo.es. 


\section{MIGRACIONES TRASNACIONALES Y GÉNERO. LA FEMINIZACIÓN COMO FACTOR CLAVE}

Vivimos en un mundo marcado por la globalización, habiéndose convertido en un elemento esencial en el análisis de los procesos internacionales. Así, la globalización, de acuerdo con Entrena (2010), también repercute en las migraciones a nivel mundial y desde diferentes prismas.

El estudio de las implicaciones de la globalización es complejo, ya que, como recoge Mora (2008), incluye diversos fenómenos socioculturales que no poseen fácil explicación, suponiendo transformaciones globales relacionadas con la trasnacionalización mercantil y económica. Es por ello por lo que son múltiples las causas y consecuencias que conforman los procesos de globalización, tales como las posibilidades de los medios de comunicación y la movilidad, las redes sociales y de apoyo, la transferencia del conocimiento, la mundialización laboral o las contingencias de formación en otros países, entre otros (Piqueras, 2012).

Centrándonos en los procesos migratorios trasnacionales, a medida que la globalización se ha extendido a lo largo del siglo $\mathrm{XX}$, la cuestión migratoria se complejiza, creándose nuevos espacios de emisión, tránsito y/o recepción, incrementándose, además, el número de migrantes, cuantitativa como cualitativamente. Este hecho ha tenido consecuencias, además, en la evolución sufrida en la tipología de las migraciones, ampliándose las causas que conducen a millones de personas a iniciar procesos migratorios. Ya no son solo flujos de personas que se mueven por motivos de trabajo y/o supervivencia, sino que se ha extendido a otras situaciones: búsqueda de nuevas formas de empleo, redes sociales, calidad de vida y estatus social, a la misma vez que pueden aparecer causas relacionadas con el alejamiento de contextos belicosos y situaciones políticas peligrosas, así como desastres medioambientales que provocan realidades de extrema pobreza. (Piqueras, 2012).

El informe publicado por la Comisión Global de Migraciones Internacionales (2005), manifestaba que las migraciones se estaban convirtiendo en una estrategia de supervivencia empleada por personas que tratan de huir de conflictos armados, gobiernos autoritarios y corruptos, violaciones de derechos, desempleo y pobreza. A este contexto debe sumarse que, en el escenario actual, los flujos migratorios se desarrollan en contextos receptores que han dejado de ser liberales para convertirse en entornos crecientemente controlados (Alba, 2009).

En este marco contextual, la presencia creciente de las mujeres en las migraciones internacionales, cobra especial interés la inclusión de una perspectiva de género que visibilice situaciones de vulnerabili- 
dad y riesgo que explican las características propias de este colectivo.

[...] La expansión a partir, sobre todo, de mediados de los noventa del siglo pasado, de los análisis relativos a la feminización de las migraciones o a la entronización de las mujeres pioneras, en tanto que agentes que inician la red migratoria de otros familiares, solas o acompañadas, y no sólo como figuras secundarias de la reagrupación familiar, no implica, en consecuencia, todavía la conceptualización plena de estos flujos como migraciones generizadas, pero, sin duda, contribuye a la emergencia de esta perspectiva (Barañano y Marchetti, 2016, p.13).

Partiendo de estas premisas, en este trabajo se realiza una revisión teórica acerca de las implicaciones que tiene la feminización de las migraciones en el contexto migratorio trasnacional, destacando la necesidad de fomentar la inclusión de esta perspectiva de género en la puesta en marcha de las acciones políticas internacionales, que redunde en una mejora de la atención a las vulnerabilidades y riesgos a los que se exponen por el solo hecho de ser mujeres.

De acuerdo con los datos aportados por la Organización Internacional para las Migraciones (2019), las mujeres representaban en 2017 un 48,4 \% de la población mundial de migrantes internacionales, cifra que ha ido aumentando desde los años sesenta. Esta feminización de las migraciones se entiende no solo como un incremento del número de mujeres que participan de los procesos migratorios, sino que constituye un proceso que tiene unas características propias que lo diferencia de las migraciones masculinas, donde las mujeres asumen un rol como líderes de sus proyectos migratorios (Rebolledo y Rodríguez, 2014; Cárdenas-Rodríguez, Terrón-Caro y Vázquez-Delgado, 2016).

Tradicionalmente, las mujeres habían sido consideradas como acompañantes de los hombres o como parte de las reunificaciones familiares, siendo los hombres los protagonistas del proyecto migratorio. Esta invisibilización de las mujeres en los flujos migratorios no es ajena a la que se manifiesta en el conjunto de la vida económica, política y social a nivel mundial como resultado de su exclusión, sujeción y subvaloración (Roldán, 2010; 2011).

Los estudios sobre migraciones comienzan a incluir la representación de las mujeres con independencia de los hombres a partir de los años setenta (Morokvasic, 1984, citado en Cruz y Rojas, 2000), si bien, desde los años ochenta, la perspectiva de género cobra sentido, interés e importancia en los estudios relacionados con las migraciones. Así, el género se convierte en categoría de análisis en multitud de investigaciones, permitiendo introducir elementos diferenciadores 
entre hombres y mujeres, entre los factores y procesos con base en oportunidades y derechos (construidos o legales) distintos en cada caso.

De esta forma, se evidencia una clara relación entre migraciones y género.

[...] Desde esta perspectiva es interesante entender cómo los procesos migratorios podrían empoderar o desapoderar especialmente a las mujeres, quienes tradicionalmente han ocupado posiciones de subordinación dentro de una cultura patriarcal, pero también cómo las relaciones de género, construidas socialmente, han dado forma a los procesos migratorios actuales (Cueva-Luna y Terrón-Caro, 2014, p.10).

La globalización, asimismo, ha profundizado las brechas entre géneros, así como entre regiones y grupos socioeconómicos. Esto se corresponde con un enfoque interseccional de la categoría de género que, de acuerdo con Roldán (2010), no se presenta como un monolito, ya que está ineludiblemente vinculada con otras estructuras sociales de desigualdad, como son la clase, edad, nacionalidad y etnia, entre otras. Dicho enfoque interseccional posibilita tener presentes las distinciones que existen, evitando la cosificación entre las mujeres migrantes, construyéndose como una categoría única (Barañano y Marchetti, 2016).
Así, las mujeres han ido asumiendo un rol más protagonista y activo en los procesos migratorios, que ha sido esencial para la comprensión de su evolución en los últimos tiempos (Rebolledo y Rodríguez, 2014). Tal y como exponen Roldán, Castro y Sarmiento (2012), esta feminización de las migraciones resalta que las mujeres comenzaron a emigrar por derecho propio y en busca de sus objetivos particulares, entre los que se pueden encontrar el deseo de mejorar su situación social y económica.

Los efectos de las transformaciones en el papel de las mujeres en las sociedades actuales han modificado también las migraciones femeninas. Izcara (2017) añade la influencia de las modificaciones en las distribuciones ocupacionales, caracterizadas por el descenso de tareas de producción y construcción frente al aumento de actividades relacionadas con la reproducción social. De la misma forma, este análisis diferenciador a causa del género se ha redimensionado a partir de la consideración de los aspectos como la familia como estrategia de los proyectos de viajes (Cárdenas-Rodríguez, Terrón-Caro y Vázquez-Delgado, 2016).

De acuerdo con Fernández-Hawrylak y Heras (2019), se constatan transformaciones profundas en las familias, produciéndose un reacomodo de las relaciones de género y generacionales, dando lugar a la negociación de los vínculos dentro del grupo doméstico y a diferentes modalidades de reagrupaciones familiares, tanto en el lugar de origen como de destino. 
“[...] la literatura revela que cada vez son más frecuentes los hogares transnacionales configurados por mujeres autónomas que han abandonado sus países, dejando en ellos a sus hijos con el esposo [...]. (Fernández y Heras, 2019, p.35).

En definitiva, de acuerdo con Binazzi (2019) las migraciones trasnacionales continúan siendo un hecho sociocultural en el que existen diferentes tipos invisibilizadas de violencia. Este hecho, por su carácter clandestino, así como por los limitados datos, evidencian la necesidad de seguir realizando investigaciones sobre esta temática desde la perspectiva de género.

\subsection{El perfil de las mujeres migrantes: el caso de México}

Entre los lugares con alta presencia de migraciones trasnacionales podemos resaltar a México, como uno de los países de origen, tránsito y destino con mayor relevancia en el continente americano, sobre todo por la realidad alrededor de la frontera con Estados Unidos. Es por ello por lo que lo hemos tomado como referencia para tratar las características de las mujeres que se sustraen de las investigaciones realizadas, pues de acuerdo con el estudio de Binazzi (2019), la frontera sur de México constituye una realidad invisible y un contexto de flujo migratorio numeroso y bastante duro.

[...] Aunque no exista todavía un mecanismo de monitoreo y reco- lección de datos sistemático, según el estudio pionero desarrollado por $\mathrm{CMDPDH}^{1}$ (2014), el fenómeno del desplazamiento interno producido por la violencia se está volviendo un drama de grandes proporciones en México. (CMDPDH, 2018, citado en Binazzi, 2019, p.165).

Las mujeres migrantes son mujeres jóvenes, con edades entre veinte y treinta años, siendo significativo que suelen iniciar las migraciones en edades más tempranas que los hombres (Woo, 1997; Vidal et al., 2002; Mendoza, 2005; 2006; Díaz y Kuhner, 2007; Cueva-Luna y Terrón-Caro, 2014).

En el contexto mexicano, la situación administrativa de las mujeres cuando migran toma especial relevancia. De acuerdo con Vidal et al. (2002), un alto porcentaje de mujeres migrantes se encuentra en condición de indocumentación, hecho que se agrava por una mayor vulnerabilidad para enmarcarse en la clandestinidad, potenciando así la explotación y extorsión de las mujeres por parte de grupos de organización criminal, autoridades, prestadores de servicio, empresarios... (París et al., 2010; Cárdenas-Rodríguez, Terrón-Caro y Vázquez-Delgado, 2016).

\footnotetext{
1 La CMDPDH es una organización civil, secular, autónoma e independiente, fundada en 1989, con estatus consultivo de la Organización de los Estados Americanos (OEA) y de la Organización de las Naciones Unidas (ONU), entre otras membresías. Véase enlace: http://cmdpdh.org/
} 
Esta vulnerabilidad se convierte en una característica propia de las personas que migran de forma indocumentada, ya que, de acuerdo con la Comisión Nacional de los Derechos Humanos de México (CNDH, 2007), es por su situación irregular por la que temen ser deportadas a su país de origen, ya que no pueden acudir a denunciar a las autoridades para exigir la protección a la que tienen derecho (citado en Morales, 2010). Esta vulnerabilidad se agrava por una cuestión de género, incrementándose los riesgos a las que las mujeres se exponen durante los procesos migratorios.

Si bien Vidal et al. (2002), también recogen casos, aunque en menor medida, en los que las mujeres inician su proyecto migratorio con su situación administrativa en orden, como pueden ser las migraciones temporales para trabajar en empleos concertados con anterioridad, suelen realizarse con una documentación administrativa reglada.

Otra de las características destacadas del perfil de las mujeres migrantes es la referente a su estado civil, siendo en su mayoría mujeres casadas y con hijos e hijas a su cargo o que inician su migración con su hijo o hija, llegándose en ocasiones a pérdidas mortales de los y las menores por el trayecto (Woo, 1997; Woo y Moreno, 2002; Vidal et al., 2002; Cárdenas-Rodríguez, Terrón-Caro y Vázquez-Delgado, 2016). Sin embargo, en otros estudios se destaca un incremento de mujeres solteras (Woo y Moreno, 2002), número que se espera vaya aumentándose gradualmente en el futuro.

Con respecto a la formación de las mujeres a la hora de migrar, estas poseen un alto nivel educativo respecto a los hombres, superando en muchas ocasiones los estudios primarios y, en menor medida, los secundarios (Woo, 1997; Ávila et al., 2000; Woo y Moreno, 2002; Vidal et al., 2002; Cárdenas-Rodríguez, Terrón-Caro y Vázquez-Delgado, 2016). No obstante, esto no se vincula directamente con una mayor experiencia en cuestiones de migración, es decir, suele persistir bastante desconocimiento sobre lo que supone iniciar un proyecto de viaje a nivel económico, de estrategias de rutas, de situaciones de riesgos y vulnerabilidad de derechos a todos los niveles, etc.

En último lugar, resaltamos la multiplicidad de motivos por los que las mujeres inician sus procesos migratorios. Teniendo en cuenta el perfil descrito de las mujeres migrantes en el contexto mexicano y, en relación con las características socioeconómicas de los países de origen, podemos distinguir tres grupos principales de causas: la situación en la que se encuentra su lugar de procedencia, su situación familiar, y las causas y circunstancias socioeconómicas (Cruz y Rojas, 2000; Monzón, 2006; Díaz y Kuhner, 2007).

Como exponen Cárdenas-Rodríguez, Terrón-Caro y Vázquez-Delgado (2016), 
las mujeres que emigran lo hacen con la convicción de insertarse en un mercado laboral donde tengan mayores oportunidades y trabajos mejor remunerados con el fin de ayudar a sus familias. Así, los proyectos migratorios femeninos se basan en la búsqueda de mejores condiciones vitales y familiares, además del interés por proporcionar una mejor educación para sus hijos e hijas y una mejora en su situación laboral y económica.

Todo ello, pese a la situación en la que pueden encontrarse, ya que el hecho de ser mujer en un contexto migratorio históricamente violento hace que las mujeres migrantes se encuentren expuestas a mayores vulnerabilidades y riesgos para convertirse en víctimas de violaciones, maltratos, secuestros y abusos de diferentes índoles (Rebolledo y Rodríguez, 2014).

A esta situación se suma la inserción en un mercado laboral donde tienen más posibilidades de ocupar trabajos considerados precarios, siendo esencial considerar

[...] los riesgos y abusos (que han cobrado la calidad de "naturales", tanto en los procesos migratorios como en su incorporación a la población económicamente activa), para estar en condiciones de precisar los niveles de vulnerabilidad en los que se desenvuelve este fenómeno (Roldán, Castro y Sarmiento, 2012, p.89).

\section{DERECHOS HUMANOS}

Y MIGRACIONES FEMENINAS:

APUNTES PARA EL FUTURO

Las migraciones son consideradas un derecho para todas las personas, en tanto hombres y mujeres emprenden un proceso migratorio con el objetivo de mejorar su calidad de vida. Concretamente, en la Declaración Universal de los Derechos Humanos se reconocen reiteradamente distintos derechos de las personas en relación con las migraciones. En su artículo 13.1, se reconoce el "derecho que toda persona tiene a circular libremente y a elegir su residencia en el territorio de un Estado", así como que "toda persona tiene derecho a salir de cualquier país, incluso del propio, y a regresar a su país" (artículo 13.2). Siguiendo a Morales (2010), las migraciones se justifican y avalan como una facultad de subsistencia y mejora de vida, además de como condición humana y un derecho como tal.

En esta misma línea, en el Preámbulo de dicha Declaración Universal se expone el valor esencial de hombres y mujeres, proyectado en un "reconocimiento de la dignidad intrínseca y de los derechos iguales e inalienables de todos los miembros de la familia humana", y por consiguiente, se hace referencia al principio de igualdad y dignidad "sin distinción alguna de raza, color, sexo, idioma, opinión política o de cualquier índole nacional o social, posición económica, nacimiento o 
cualquier otra condición” (artículo 2).

En último término, la Declaración Universal de los Derechos Humanos promueve la igualdad de trato y los derechos entre individuos nacionales y extranjeros. Sin embargo, en su artículo 13, como apunta Bonet (2002) "no incluye ni ampara el derecho de cualquier persona a entrar libremente en otro país que no sea el suyo" (citado en Morales, 2010, p.22).

A tenor de lo expuesto sobre los derechos humanos, dicha declaración al referirse a la igualdad de género,

[...] hace patente el mantenimiento de la consistencia conceptual con la implementación de políticas públicas que tienen que aplicar todos los instrumentos y medidas para que se concrete una real igualdad entre mujeres y hombres (Binazzi, 2019. p.170).

Si bien los procesos migratorios femeninos son foco de interés de distintas instituciones e investigadores/as, se debe seguir profundizando en la sistematización y transparencia en la publicación de datos, incluyendo los testimonios de las propias mujeres, de manera que se visibilice y difunda el contexto al que se enfrentan. En este sentido, resaltamos el compromiso global de la comunidad internacional de la Agencia para el Desarrollo Sostenible 2030 y de sus Objetivos (ODS), aprobada por la ONU en 2015, en la que se prioriza entre sus objetivos la igualdad de género y erradicación de toda forma de violencia de género, en su objetivo número 5 .

Así, entre las entidades que pretenden dar respuesta a las necesidades y especificidades de las mujeres migrantes, haciendo especial hincapié en el ámbito de la protección y seguridad antes, durante y después de sus viajes migratorios, podemos destacar la Organización Internacional de las Migraciones (OIM), la mencionada Organización de las Naciones Unidas (ONU), la Comisión Interamericana de Derechos Humanos (CIDH), Amnistía internacional, el Observatorio Permanente de la Inmigración (OPI), la Convención Internacional sobre la Protección de los Derechos de todos los trabajadores Migratorios y de sus familias, la Organización Internacional del Trabajo para las Migraciones Laborales, y un sinfin de organizaciones nacionales e internacionales que luchan por visibilizar y mejorar la situación de las mujeres en las migraciones.

Partiendo de lo planteando en Rebolledo y Rodríguez (2014), y ampliando su propuesta, podemos apuntar algunas líneas de actuación ante estas problemáticas:

- La renovación e implementación de las obligaciones asumidas en documentos internacionales y nacionales de carácter oficial sobre los Derechos Humanos, así como la potenciación de iniciativas de organizaciones de 
mujeres y centros de investigación de estudios superiores que trabajan en esta materia, haciendo hincapié en las migraciones femeninas.

- El análisis de textos oficiales en materia de protección de las mujeres, teniendo en cuenta los Derechos $\mathrm{Hu}-$ manos, la erradicación de toda forma de violencia sobre las mujeres y la atención al fenómeno migratorio femenino por medio de estrategias de coordinación interinstitucional.

- La creación de redes de sensibilización y solidaridad interconectadas en lo local, regional y nacional, con el fin de dar a conocer a la población la problemática actual de las mujeres migrantes, sobre las causas y consecuencias de la migración y establecer estrategias comunitarias para la protección de sus derechos.

- La defensa y el apoyo de una administración de justicia que eviten procedimientos que impiden el acceso de las mujeres a recursos judiciales y medios de protección eficaces a la hora de denunciar situaciones de vulnerabilidad de sus derechos e intimidad durante los procesos migratorios.

- La difusión de investigaciones y buenas prácticas en relación con las migraciones desde una perspectiva de género, así como la denuncia y visibilización de las situaciones de vulnerabilidad y riesgos que sufren las mujeres en los procesos migratorios.

- El trabajo conjunto con las autorida- des para fomentar programas y proyectos de concienciación sobre las migraciones, los Derechos Humanos, las políticas sociales y la seguridad ciudadana, atendiendo a las características propias de las migraciones femeninas.

- La unión de diferentes entidades políticas y sociales en la denuncia de la criminalización en los procesos migratorios, especialmente femeninos, en términos de agresiones físicas, sexuales y psicológicas, corrupción y la persistencia de la delincuencia organizada.

En definitiva, se hace necesaria una política coordinada que actúe de forma integral sobre la realidad de las migraciones femeninas, luchando contra las discriminaciones a causa del género, así como que se trabaje por la desaparición de los riesgos y vulnerabilidades que sufren las mujeres migrantes cuando deciden iniciar un proyecto de viaje que busca la mejora de su bienestar y el de sus familias.

\section{REFERENCIAS BIBLIOGRÁFICAS}

Alba, F. (2009). Migración internacional y políticas públicas. En P. Leite y S. Giourguli, Las políticas públicas ante los retos de la migración mexicana a Estados Unidos. México: Consejo Nacional de Población.

Ávila, J.L., Fuentes, C. y Tuirán, R. (2000). Mujeres mexicanas en la migración a Estados Unidos. En R. Tuirán (Coord.), Migración México-Estados Unidos. 
Continuidad y cambio. México: Consejo Nacional de Población.

Barañano Cid, M., y Marchetti, S. (2016). Perspectivas sobre género, migraciones transnacionales y trabajo: rearticulaciones del trabajo de reproducción social y de cuidados en la Europa del Sur. Investigaciones Feministas, 7(1), 9-33.

Binazzi, A. (2019). Género y migraciones forzosas en Centroamérica y el Caribe. Una mirada antropológica para los derechos humanos de las niñas, adolescentes y de las mujeres. Collectivus, Revista de Ciencias Sociales, 6(1), 155-176.

Cárdenas-Rodríguez, R. Terrón-Caro, T. y Vázquez-Delgado, B. (2016). Migraciones femeninas: Mujeres latinoamericanas indocumentadas rumbo a Estados Unidos. Revista Guillermo de Ockham. 14(2), 65-72.

Comisión Global de Migraciones Internacionales (octubre de 2005). Migration in an Interconnected World: New Directions for Action, Report of the Global Commission on International Migrations.

Cruz, H. y Rojas, M. (2000). Migración femenina internacional en la frontera sur de México. Papeles de Población, 23, 127151.

Cueva-Luna, T.E. y Terrón-Caro, T. (2014). Vulnerabilidad de las mujeres migrantes en el cruce clandestino por Tamaulipas-Texas. Papeles de Población, 79, 209-241.

Díaz, G. y Kunher, G. (2007). Globalización y migración femenina. Experiencias en México. CEPI Working Paper, 12, 1-38.

Entrena Durán, F. (2010). Impactos de las migraciones. Especial referencia a sus efectos educativos. Tempora, 13, 13-37.
Fernández-Hawrylak, M. y Heras Sevilla, D. (2019). Familias transnacionales, familias inmigrantes: Reflexiones sobre su inclusión en la escuela. Revista de Sociología de la Educación, 12(1), 24-39.

Izcara Palacios, S.P. (2017). Feminización de la migración. Tráfico de migrantes y trata en México. Revista Electrónica Iberoamericana, 11(1), 1-17.

Mendoza, C. (2005). La migración femenina en el contexto de cambio de patrón migratorio México-Estados Unidos: Aportes de cuatro encuestas mexicanas de los noventa. Mobilités au feminin, Tánger, 15-19-Nov-2005.

Monzón, S. (2006). Las viajeras invisibles: mujeres migrantes en la región centroamericana y el sur de México. Guatemala: PCS-CAMEX.

Mora, C. (2008). Globalización, Género y Migraciones. Revista Polis, 7(20), 285-297.

Morales, J. (2010). Violencia y derechos humanos de centroamericanos en México: la paradoja entre ser un país de migrantes y un país de tránsito. Boletín Americanista, Año LX(60), 227-246.

ONU. (1948). Declaración Universal de los Derechos Humanos. Disponible en https://www.hchr.org.co/documentoseinformes/documentos/carceles/1_ Universales/B\%E1sicos/1_Generales_ DH/1_Declaracion_Universal_DH.pdf Organización Internacional para las Migraciones (2019). Gender and Migration. Recuperado de https://migrationdataportal.org/themes/gender

París, M.D. et al. (2010). Coloquio sobre Derechos Humanos y vulnerabilidad de los migrantes y repatriados en la frontera 
norte de México. El Colegio de la Frontera Norte, Tijuana, Baja California, 2 y 3 de diciembre de 2010.

Piqueras Haba, J. (2012). El mundo en movimiento. Migración internacional y globalización. Cuaderno de Geografía, 90, 187-210.

Rebolledo Gámez, T. y Rodríguez Casado, M. R. (2014). Migraciones y género en el contexto mexicano: revisión de la literatura científica. Foro de Educación, 12(17), 165-185.

Roldán, G. (2010). Derechos Humanos y Migración. En N. Pérez (Coord.), Hacia la Construcción de políticas públicas a favor de las mujeres migrantes. Caso Chiapas. México: INCIDE Social, Unión Europea y Sin Fronteras IAP.

Roldán, G. (2011). Acciones y políticas internacionales a favor de las mujeres migrantes. En N. Pérez y G. Roldán, Mujeres migrantes en el Soconusco. Situación de su derecho a la salud, la identidad y al trabajo. México: INCIDE Social.
Roldán, G., Castro-Alquicira, D. y Sarmiento, A.L. (2012). Hacia la construcción de políticas públicas a favor de las mujeres migrantes. El caso de Chiapas en México. Dilemata, año 4(10), 85-118.

Vidal, L., Tuñón, E., Rojas, M. y Ayús, R. (2002). De Paraíso a Carolina del Norte. Redes de apoyo y percepciones de la migración a Estados Unidos de mujeres tabasqueñas depulpadoras de jaiba. $M i-$ graciones Internacionales, 1(2), 1-34.

Woo, O. (1997). Migración femenina indocumentada. Frontera Norte, 9(17), 113-129. Woo, O. y Moreno, J. (2002). Las mujeres migrantes y familias mexicanas en Estados Unidos. Migración: México entre sus dos fronteras, 2000-2001, 105-113.

\section{PARA CITAR ESTE ARTícULO}

Rebolledo Gámez, T. y Rodríguez Casado, R. (2019). Mujeres migrantes, vulnerabilidad y Derechos Humanos. Collectivus, Revista de Ciencias Sociales, 6(2), 59-69

DOI: http//dx.doi.org/10.15648/Coll.2.2019.4

Recibido: 15/11/2018 Aprobado: 16/02/2019 